\title{
Marietta Meier
}

\section{Auf der Kippe: Spannungskonzepte an der Wende vom 19. zum 20. Jahrhundert}

Spannung bezeichnet einen Zustand auf der Kippe: eine Zeitspanne, während der offen ist, welchen Weg etwas gehen wird, einen statischen, aber instabilen Zustand am Rande produktiver oder destruktiver Prozesse. ${ }^{1}$ Während der langen Jahrhundertwende, womit im Folgenden die Zeit zwischen 1880 und 1930 gemeint ist, findet sich der Begriff Spannung in unterschiedlichen Kontexten und Bedeutungen. Von Spannungen war in der Kunst, im Alltag, in der Politik und in verschiedenen Wissenschaftsdisziplinen die Rede. Spannung war ein boundary concept, ein unscharfer Begriff, der zwischen verschiedenen Bereichen zirkulierte. ${ }^{2}$ Im Zuge dieser ,Reise' wurden die Grenzen zwischen den unterschiedlichen Bedeutungen des Begriffs erweitert und verwischt, es kam zu Übersetzungs- und Aneignungsprozessen.

Im folgenden Beitrag wird der Weg rekonstruiert, den der Begriff Spannung im Laufe des 19. Jahrhunderts im deutschsprachigen Raum ging. ${ }^{3}$ Untersucht wird, in welchen Zusammenhängen der Begriff verwendet wurde, was er im jeweiligen Kontext bedeutete, wie sich der Begriff durch den Transfer von einem Feld zum anderen veränderte und wie sich die verschiedenen Spannungskonzepte gegenseitig beeinflussten. Im Zentrum steht die Psychiatrie, die sich ab den 1860er Jahren als akademische Disziplin etablierte und eine Expertenrolle übernahm, die weit über den Auftrag zu heilen und zu verwahren hinausreichte.

Das Spannungskonzept, das die Psychiatrie um 1900 vertrat, widerspiegelt - so die These - verschiedene Dimensionen des Spannungsfelds, in dem diese Disziplin an der Wende vom 19. zum 20. Jahrhundert stand. In der Psychiatrie ging es um unterschiedliche Formen von Spannung, deren Ursachen und Folgen sowie um die Frage, wann Spannung pathologisch oder auf psychische Störungen zu-

\footnotetext{
${ }^{1}$ Vgl. Christoph F. E. Holzhey (Hg.): Tension/Spannung. Wien/Berlin 2010. Der Sammelband entstand in Zusammenhang mit dem Projekt des ICI Berlin Institute for Cultural Inquiry zum Thema „Spannung“.

2 Ilana Löwy: The Strength of Loose Concepts. Boundary Concepts, Federative Experimental Strategies and Disciplinary Growth. The Case of Immunology. In: History of Science 30 (1992), S.371-396; dies.: Unscharfe Begriffe und föderative Experimentalsysteme. Die immunologische Konstruktion des Selbst. In: Hans-Jörg Rheinberger/Michael Hagner (Hg.): Die Experimentalisierung des Lebens. Experimentalsysteme in den biologischen Wissenschaften 1850/1950. Berlin 1993, S. 188-206.
}

${ }^{3}$ Vgl. Mieke Bal: Travelling Concepts in the Humanities. A Rough Guide. Toronto 2002. 
rückzuführen sei. Als boundary concept ermöglichte der Begriff, an ein weitverbreitetes Alltagsverständnis von Spannung anzuknüpfen, soziale Allianzen zu bilden und zwischen verschiedenen Wissensfeldern und Wissenschaftskulturen zu interagieren. Die Psychiatrie verwendete den Ausdruck deshalb als Scharnier, das naturwissenschaftliche Prinzipien mit subjektiven Gefühlen und zwischenmenschlichen Beziehungen verband, ohne dass geklärt werden musste, wie diese miteinander zusammenhingen. Auf diese Weise entstand ein Spannungskonzept, das Spannung zunehmend mit zielgerichteter Kraft, Eruption und Gefahr assoziierte.

\section{Spannen, Dehnen und Erwarten: Ein etymologischer Überblick ${ }^{4}$}

Der Begriff Spannung, der im Deutschen ab dem 17. Jahrhundert belegt ist, entwickelte sich schon aufgrund seiner Etymologie zu einem äußerst vieldeutigen Ausdruck. Spannen beziehungsweise Spannung haben vermutlich eine gemeinsame Wurzel mit den griechischen Wörtern span für ziehen, zerren beziehungsweise spasmos für Krampf. Die fachsprachlichen Begriffe Tonus und Tension, das französische tension und das englische tension gehen hingegen auf lateinisch tonus beziehungsweise griechisch tonos für Spannung zurück, aus denen auch Ton entlehnt ist. Analog zum transitiven Gebrauch des Verbs spannen, das bereits im Mittelhochdeutschen verwendet wurde, bezog sich Spannung zunächst ausschließlich auf die Tätigkeit des Spannens oder den Zustand des Gespanntseins: Gespannt wurden und waren Pferde, Bogen oder Saiten. So führt Zedlers „Universallexikon", das in der ersten Hälfte des 18. Jahrhunderts erschien, die Begriffe Spannen, Anspannen, Abspannen und Ausspannen auf. Unter Spannung findet sich neben der "Spannung des männlichen Gliedes“ nur ein Verweis auf Ausspannung - einen Begriff aus der Naturlehre, der den Zustand von „Cörper[n]“ bezeichnete, die „nach ihrer Länge vergrössert [...] und unterdessen in der Breite und Höhe" verkleinert wurden. ${ }^{5}$

${ }^{4}$ Dieser Überblick beruht auf einschlägigen Artikeln in verschiedenen Wörterbüchern: Gespannt. In: Deutsches Wörterbuch von Jacob und Wilhelm Grimm, Bd.5 [Originalzählung IV, 2,3]. Leizpig 1897, Sp.4135; Spannung. In: Deutsches Wörterbuch von Jacob und Wilhelm Grimm, Bd.16 [Originalzählung X, 1]. Leipzig 1905, Sp.1915f.; Digitales Wörterbuch der Deutschen Sprache, Etymologisches Wörterbuch, http://www.dwds.de/; Entspannung. In: Historisches Wörterbuch der Philosophie. Hg. von Joachim Ritter/Karlfried Gründer, Bd. 2. Basel/Stuttgart 1972, Sp. 548-550; Spannung. In: Historisches Wörterbuch der Philosophie. Hg. von Joachim Ritter/Karlfried Gründer, Bd. 9. Basel 1995, Sp. 1282-1300. Sofern nicht anders vermerkt, beruhen die Aussagen zur Bedeutung und Verwendung des Begriffs Spannung auf den Ergebnissen einer Recherche im Kernkorpus des Deutschen Textarchivs (ca. 1600 bis 1900) und sämtlichen weiteren Korpora des Digitalen Wörterbuchs der Deutschen Sprache, die im April/ Mai 2014 durchgeführt wurde. Für Informationen zu diesen Korpora siehe http://www.dwds.de/ ressourcen/korpora/.

5 Ausspannung. In: Johann Heinrich Zedler: Grosses vollständiges Universallexikon, Bd. 2. Halle a. d. S./Leipzig 1732, Sp. 2250; Spannen, Spannung. In: Johann Heinrich Zedler: Grosses vollständiges Universallexikon, Bd. 38. Halle a. d. S./Leipzig 1743, Sp. 1205, Sp. 1207. 
Im Laufe des 18. Jahrhunderts begann man, auch von einer Spannung des Gemüts oder der Seele zu sprechen. Neu konnte Spannung zudem einen Zustand gestörten Einvernehmens meinen. In Adelungs „Wörterbuch der Hochdeutschen Mundart“ von 1801 findet sich unter Spannung der Satz „sie sind miteinander gespannt“; ein „figürliche[r]“ Ausdruck, den man - wie erklärt wird - für „Personen“ verwende, die "nicht in dem besten Vernehmen mit einander stehen, ohne eben Feinde zu seyn". ${ }^{6}$ Auf der politischen und der gesellschaftlichen Ebene waren Spannungen in der Regel unerwünscht; es galt zu verhindern, dass Spannungen entstanden, wuchsen oder gar in einen gewalttätigen Konflikt mündeten.

$\mathrm{Ab}$ dem 19. Jahrhundert konnte Spannung schließlich auch den psychischen Zustand eines Menschen meinen, der etwas erwartete, dessen Aufmerksamkeit erregt war. Besonders deutlich wird der Zusammenhang von Spannung und attentio in der Musiktheorie, der Ästhetik, der Dichtung und der Rhetorik, wo Spannung als bewusst erzeugtes, intentionales Mittel verstanden wurde. Hier meinte Spannen seine Aufmerksamkeit auf etwas richten, etwas genau verfolgen, war also positiv konnotiert. Evident wird diese Wertung im Adjektiv spannend, das seit dem 19. Jahrhundert belegt ist. In der Rhetorik behandelte man die intentio des Richters oder des Zuhörers, die vor allem als aufmerksame Gespanntheit verstanden wurde, in erster Linie im Zusammenhang mit der Frage, wie eine Rede zu beginnen sei. Besonders für das genus bumile, den schlichten Stil, stellte das Erlangen von Aufmerksamkeit eine elementare Zielvorgabe dar. Kunstlehren, die sich mit Wirkungsästhetik befassten, schrieben der Spannung ebenfalls eine wichtige Aufgabe zu. Dabei galt die Art der erzeugten Spannung lange als Maß für künstlerische Qualität. Kunstwerke sollten sich durch eine Spannung auszeichnen, die auf die „angespannte Erwartung des Gemüts“ abzielte. So heißt es beispielsweise in einem kurzen Text über Spannung, der 1909 in der Zeitschrift „Kunstwart“ erschien: „Je weniger die Spannung eines Dichtwerks mit Neugier zu tun hat, um so reiner, um so echter ist es in künstlerischem Betracht.“ Nicht „äußere“ Spannung sollte also geweckt werden, sondern Spannung „aus Teilnahme“, die sich durch „Bewegung in irgendeiner ihrer unendlich vielen Formen (Handlung, Gang, Rhythmus, Licht, Wille)“ erzeugen ließ.7

Im Gegensatz zur Politik oder Ästhetik hatte der Begriff Spannung in den Naturwissenschaften an sich keine wertende Bedeutung. In der Physik kam der Ausdruck im Sinn von Ausdehnung zum Tragen. Hier war Spannung zunächst die Kraft im Inneren eines elastischen Körpers, also mechanische Spannung. Das Konzept der Elastizität bildete den Hintergrund für die Versuche zur „Spannkraft von Dämpfen“, die Mitte des 18.Jahrhunderts erstmals durchgeführt und drei Jahrzehnte später in großem Umfang vorgenommen wurden. Mit der Aufstellung der „Spannungs-Reihe der Metalle“ und der Entdeckung des SpannungsGesetzes durch Alessandro Volta gewann dieser physikalische Begriff von

\footnotetext{
${ }^{6}$ Spannen. In: Johann Christoph Adelung: Grammatisch-kritisches Wörterbuch der Hochdeutschen Mundart, Bd. 4. Leipzig 1801, Sp. $162 \mathrm{f}$.

7 Willy Rath: Von der Spannung. In: Der Kunstwart 32 (1909), S. 159-162, hier: S. 159, S. 162.
} 
Spannung seit dem 19. Jahrhundert eine neue Bedeutungsschicht. Nun wurde Spannung auch und schließlich in erster Linie als elektrische Spannung verstanden. Elektrische Spannung entsteht, wenn zwei gegensätzlich geladene Körper, die aufgrund ihrer gegenseitigen Anziehung miteinander verbunden sind, voneinander entfernt werden. Um die Körper zu trennen, muss eine gewisse Kraft aufgewendet werden. Diese Kraft wird im Zustand der Spannung gleichsam aufbewahrt. Als eine Art „Kraftpotential“ kann die elektrische Spannung in einem Leiter Elektronen in Bewegung setzen, also Strom fließen lassen, der sich in Energie umsetzen lässt.

\section{Spannung oder Tonus? Medizinische Terminologie}

Wie in der Physik steht Spannung auch in der Medizin mit dem Begriff Kraft in Zusammenhang. Wurde hier unter Spannung zunächst der Spannungszustand eines Muskels oder Gewebes verstanden, war ab dem 18. Jahrhundert auch von einer Spannung des Gemüts oder der Seele die Rede. Johann Christian Reil, der prominenteste Vertreter der deutschen romantischen Medizin, spricht in seinen „Rhapsodieen über die Anwendung der psychischen Curmethode auf Geisteszerrüttungen“ von 1803 beispielsweise von einer Spannung der Seele und der Fantasie. ${ }^{8}$ Der medizinische Fachbegriff Tonus bezog sich hingegen auf körperliche Spannungszustände. Tonus bezeichnete den Grad der Spannung, den ein lebendes Gewebe, Organ oder Organteil aufwies; er konnte hoch oder niedrig sein, musste aber immer aufrechterhalten bleiben. So erwähnt Albrecht von Haller in der zweiten Hälfte des 18. Jahrhunderts, dass Opium „den Tonus des Magens“ beeinträchtige. An einer anderen Stelle verwendet er zwar den Begriff Spannungskraft, fügt jedoch in Klammern den lateinischen Ausdruck Tonus an. ${ }^{9}$

Mit der Ausbildung der klinischen, naturwissenschaftlich ausgerichteten Psychiatrie verschwanden die Seele und deren Spannungen aus dem Blickfeld. Wilhelm Griesinger verwendete in seiner „Pathologie und Therapie der psychischen Krankheiten“ von 1845 zwar den Ausdruck Spannung, brachte ihn aber nur mit körperlichen Vorgängen in Verbindung, sprach also beispielsweise von einer Spannung der Hirnhaut. Gleichzeitig prägte er den Begriff des psychischen Tonus, den er mit dem körperlichen Tonus verglich. Unter psychischem Tonus verstand der deutsche Psychiater „das erworbene mittlere Maß psychischer Kraft“, das er auf den Charakter eines Menschen zurückführte. Körperlicher und psychischer Tonus konnten, so Griesinger, durch Krankheiten stark verändert werden: „Es ist nicht der gewöhnliche Ausdruck, und es wäre allzuabstract [!], aber es wäre nicht unrichtig, den Tetanus, die Convulsionen etc. als Abänderungen des Tonus [...]

\footnotetext{
${ }^{8}$ Johann Christian Reil: Rhapsodieen über die Anwendung der psychischen Curmethode auf Geisteszerrüttungen. Halle a.d. S. 1803, S. 106, S. 188f., S. 204, S. 267.

9 Albrecht von Haller: Anfangsgründe der Phisiologie des menschlichen Körpers, Bd.2. Berlin 1762, S. 719; ebd., Bd. 5. Berlin 1772, S. 31.
} 
aufzufassen; denn unzweifelhaft leidet der Tonus hier sogleich unter der vorhandenen Störung. Ebenso ist die auffallendste Störung bei den parallelen Geisteszuständen [...] die Störung des Gemüths, und in diesem Sinn ist überhaupt von den Gemüthsleiden und ihrer Primitivität beim Irresein zu sprechen. " ${ }^{10}$ Geisteskrankheiten führten laut Griesinger entweder zu einem anhaltend tiefen psychischen Tonus, der mit einer „gehemmten Spannung“, oder einem anhaltend hohen psychischen Tonus, der mit einer „convulsivischen Erschütterung und Losgelassenheit des Strebens" einherging. ${ }^{11}$

In Griesingers Tonuskonzept vermischten sich altbekannte Denkfiguren mit neuen. Auf der einen Seite knüpfte der Psychiater an eine lange Tradition an: Das Ideal der Ausgewogenheit, des Gleichgewichts zwischen Gefühlen, Körpersäften und kosmischen Kräften findet sich schon in der Antike und blieb in Form der Humoralpathologie bis zum 18. Jahrhundert von Bedeutung. Auf der anderen Seite sprach Griesinger als Somatiker nicht mehr von Spannungen der Seele, sondern übernahm den medizinischen Fachbegriff und übertrug ihn auf die psychische Ebene. Er unterschied zwischen körperlichem und psychischem Tonus, verknüpfte aber letzteren mit dem physikalischen Begriff der Spannung, wie er in der Elektrizitätslehre und der Physiologie verwendet wurde. Diese Verbindung sollte die Psychiatrie der nächsten Jahrzehnte entscheidend prägen.

\section{Nervöse Spannungen: Nerven als Bindeglied zwischen Körper und Psyche?}

Als sich die Physiologie im zweiten Drittel des 19. Jahrhunderts als medizinische Disziplin etablierte, eröffnete die Elektrizitätslehre neue Möglichkeiten zum Verständnis des Nervensystems. Die Arbeiten von Du Bois-Reymond, Helmholtz, Duchenne, Remak und Hitzig hatten zur Folge, dass der Elektrizität in der medizinischen Forschung und bei der Behandlung des Muskel- und Nervensystems eine immer wichtigere Rolle zukam. Umgekehrt wurde das Nervensystem zunehmend als komplexes, störanfälliges System elektrischer Impulse, Leitungsbahnen und Energieströme interpretiert. ${ }^{12}$ Das mechanistische, eng mit der Industrialisierung zusammenhängende Körperkonzept verschmolz mit neuen Erkenntnissen aus der Neurologie. Es entstand ein neues Modell, das den Körper als „reizbare Maschine " konzipierte. ${ }^{13}$

Dass die „Elektrizifierung des Körperkonzepts“ auch die Psychiatrie beeinflusste, zeigt sich beispielsweise beim deutschen Psychiater Karl Kahlbaum, der

10 Wilhelm Griesinger: Die Pathologie und Therapie der psychischen Krankheiten, für Aerzte und Studirende. Stuttgart 1845, S. 43.

11 Ebd., S. 254, S. 259.

12 Volker Roelcke: Krankheit und Kulturkritik. Psychiatrische Gesellschaftsdeutungen im bürgerlichen Zeitalter (1790-1914). Frankfurt a. M./New York 1999, S.110f.

13 Philippe Sarasin: Reizbare Maschinen. Eine Geschichte des Körpers 1765-1914. Frankfurt a. M. 2001. 
1863 eine neue Gliederung psychischer Störungen aufstellte und erstmals die Katatonie als psychiatrisches Krankheitsbild definierte. Die Bezeichnung Katatonie oder Spannungsirresein gründete auf den auffälligen körperlichen Spannungen, die Kahlbaum bei einer bestimmten Gruppe von Geisteskranken beobachtet hatte und die er auf einen veränderten „Spannungzustande der Musculatur oder vielmehr der betreffenden Nerven“ zurückführte. Der Ausdruck Spannungsirresein verwies also auf einen starken Spannungszustand der Nerven, der sich in einem sichtbar erhöhten Muskeltonus manifestierte. Kahlbaum verstand die Bezeichnung allerdings ausdrücklich auf rein phänomenologischer Ebene, er wollte damit keine Aussagen über die Ursachen des Symptoms oder der Krankheit machen. ${ }^{14}$

In seiner Studie zur Katatonie von 1874 ist aber noch in einem zweiten Sinn von Spannung die Rede. So machte Kahlbaum bei katatonen Patienten, die nichts essen und trinken wollten, „eine allgemeine negative Spannung, eine Tendenz zur Negation“ aus, die „Nahrungsverweigerung“ scheine deshalb „nur ein Moment dieser allgemeinen negativen Tendenz zu sein“. ${ }^{15}$ In diesem Beispiel sprach er von einem inneren Zustand, verwendete also Spannung in einer Bedeutung, die sich im Deutschen seit dem 18. Jahrhundert findet und in der Literatur eine wichtige Rolle spielte. ${ }^{16}$ Der Psychiater ging hier scheinbar selbstverständlich von der Existenz innerer Spannungen aus, die ein Subjekt empfinden kann, anderen aber höchstens vermittelt zugänglich sind - sei es, indem jemand über seinen Zustand spricht, sei es, indem durch Mimik, Gestik oder andere Verhaltensweisen auf innere Spannungen geschlossen wird. Kahlbaum brachte also Geisteskrankheit mit körperlichen und mit psychischen Spannungen in Verbindung. Er ließ zwar einen allfälligen Zusammenhang zwischen körperlicher und psychischer Ebene offen, betonte jedoch das Zusammenspiel von äußerer und innerer Spannung, indem er darauf hinwies, dass der äußerlich erkennbare hohe Muskeltonus auf einem Spannungszustand der Nerven beruhe.

Wenige Jahre später wurden „gespannte Nerven“ zu einem Zeitsymptom. 1881 erschien „Neurasthenia“, das Buch des New Yorker Nervenarztes George M. Beard, in deutscher Übersetzung. Die Publikation gab den Anstoß zu einer großen Zahl von Neuerscheinungen, die erst kurz vor dem Ausbruch des Ersten Weltkriegs abnahm. Nach Beard stellte Nervenkraft einen treibstoffartigen Energieträger dar. „Nervenschwäche“ entstehe, wenn mehr Nervenkraft verbraucht als zugeführt

\footnotetext{
14 Karl Kahlbaum: Die Katatonie, oder Das Spannungsirresein. Eine klinische Form psychischer Krankheit. Berlin 1874, S. 23.

15 Ebd., S. 48.

16 Vgl. bspw. Thomas Anz: Spannung - eine exemplarische Herausforderung der Emotionsforschung, online zugänglich unter: http://www.literaturkritik.de/public/rezension.php?rez id=14010 (letzter Zugriff am 12.10.2015); Rupert Gaderer: Poetik der Technik. Elektrizität und Optik bei E. T. A. Hoffmann. Freiburg i. Br. 2009; Michael Gamper: Elektropoetologie. Fiktionen der Elektrizität 1740-1870. Göttingen 2009; Ingo Irsigler/Christoph Jürgensen/Daniela Langer (Hg.): Zwischen Text und Leser. Studien zu Begriff, Geschichte und Funktion literarischer Spannung. München 2008.
} 
werde. ${ }^{17}$ War Nervenschwäche um 1800 in Deutschland vor allem auf eine „Stumpfheit der Nerven“, einen Mangel an Reizen, zurückgeführt worden, wurde die Krankheit nun mit der modernen Zivilisation in Verbindung gebracht, die - so die Vorstellung - dem Menschen zunehmend Nerven- statt Muskelkraft abforderte, was zu einer Überreizung der Nerven führen konnte.

Wie der deutsche Psychiater Otto Binswanger in seinen Vorlesungen zur Neurasthenie ausführte, klagten Patienten und Patientinnen am Ende des 19. Jahrhunderts dementsprechend über ihre „aufgeregten“, „auf Drähte gespannten“, „gezerrten“ und „vibrierenden“ Nerven, die es wieder zu „beruhigen“ gelte. Binswanger berichtete, dass seine Patienten an ganz unterschiedlichen Stellen des Körpers unter „Spannungsempfindungen“ litten, die sich zum Teil objektivieren ließen, er selbst aber brachte psychische und physische Spannung nicht miteinander in Zusammenhang. ${ }^{18}$ Ähnlich ging die Psychoanalytikerin Marie Bonaparte in einem Text über den „Fall Lefebvre“ vor. Marie-Félicité Lefebvre, die Gattin eines reichen französischen Bierbrauers, brachte 1925 ihre schwangere Schwiegertochter um, weil sie - so Bonapartes Fazit - mit der Heirat des Sohns nicht einverstanden war. Der Beitrag gibt Notizen wieder, in denen Lefebvre beschreibt, wie stark sich ihre Nerven bewegten und schmerzten. Bonaparte zitiert den „vielgenannte[n]“ Text, der mit den Worten „Depression, Müdigkeit ... Neurasthenie, Unruhe, Neurose. Heirat, Kinder“ endet, in seiner ganzen Länge, fügt jedoch anschließend ironisch bei, Lefebvre gefalle sich im „Aktenstück 300 [...] in der ausführlichen Schilderung der verkrampften, gespannten und verkrümmten Nerven“. ${ }^{19}$ Weit weniger Zurückhaltung zeigten medizinische Ratgeber. So rief das „Grosse Kneippbuch“, das ab 1903 in zahlreichen Auflagen erschien, seine Leser dazu auf, sich zu beobachten und zu prüfen, ob sie ihre Arbeit mit „einer angemessenen Anspannung“ der „Nerven bzw. Körperkräfte“ erledigten. Viele Menschen würden ihre Pflichten nämlich „mit einem Übermaß an Spannungen“ verrichten und über „solche Dauerspannungszustände in einen ,überspannten Nervenzustand" hineingeraten“. 20

Mit der Durchsetzung des Elektrizitätsparadigmas begann man also, auch Alltagserfahrungen in physikalischen Kategorien zu deuten. Die Nerven wurden zum Bindeglied zwischen Körper und Geist. Neurasthenie war die Folge einer

17 George M. Beard: Die Nervenschwäche (Neurasthenia), ihre Symptome, Natur, Folgezustände und Behandlung. Leipzig 1881 (amerikanisches Original 1880).

18 Otto Binswanger: Die Pathologie und Therapie der Neurasthenie. Vorlesungen für Studierende und Aerzte. Jena 1896, S. 406.

19 Marie Bonaparte: Der Fall Lefebvre. Zur Psychoanalyse einer Mörderin. Leipzig/Wien/Zürich 1929, S. 26f. (franz. Original 1927). Lefebvre schrieb die Notizen auf die Rückseite einer Todesanzeige aus dem Jahr 1925. In ihren Heften und Notizen wiederholt sich das Motiv Nerven „unaufhörlich“. Nach Bonaparte reproduzierte Lefebvre in ihrer Hypochondrie Empfindungen aus der Zeit ihrer Schwangerschaft und Entbindung.

20 Sebastian Kneipp/Bonifaz Reile (Hg.): Das große Kneippbuch. München 1903, S. 97. Obwohl körperliche und psychische Spannung in diesem Werk miteinander in Zusammenhang gebracht werden, stehen die „überspannten Nerven“ interessanterweise auch hier in Anführungszeichen. 
allgemein verbreiteten Nervosität, betroffen waren Personen, die aufgrund von Überaktivität unter Erschöpfungszuständen litten oder ihre Spannungen zu wenig abreagieren konnten. ${ }^{21}$ Da bei der Neurasthenie die gesellschaftlichen Ursachen der Krankheit stark betont wurden, unterschied sie sich allerdings klar von den als irreversibel geltenden Geisteskrankheiten. Eine explizite Verbindung zwischen physischer und psychischer Ebene zogen Psychiater auch bei dieser Diagnose nicht.

Postuliert wurde ein solcher Zusammenhang hingegen in der Psychophysik, einem Zweig der Psychologie, die sich im ausgehenden 19. Jahrhundert als eigenständige akademische Disziplin herausbildete. Die Psychophysik zielte wie die im 19. Jahrhundert aufstrebenden Naturwissenschaften darauf ab, das überkommene Leib-Seele-Problem mit den Mitteln der empirischen Forschung zu lösen und psychische Zustände aus körperlichen Vorgängen zu erklären. ${ }^{22}$

Nach Wilhelm Wundt, der sich zu jener Zeit vor allem mit der Physiologie der Wahrnehmung beschäftigte, besaßen „Aufmerksamkeitsvorgänge“ „physiologische Substrate" und gingen stets mit Spannung und Entspannung einher. In seinen „Grundzüge[n] der physiologischen Psychologie“ kam er zu dem Schluss, es dränge sich auf, „die Perception eines Eindrucks als unmittelbar zusammenfallend mit der Erregung des zugehörigen Sinnescentrums [...] anzusehen, so dass Erregung des Rindencentrums eines Sinnesorgans und Erhebung über die Schwelle des Bewusstseins die beiden Seiten eines und desselben psychophysischen Vorgangs“ seien. ${ }^{23}$ Spannung und Entspannung erfolgten nach Wundt aber nicht nur bei Aufmerksamkeitsvorgängen, sondern etwa auch im Zusammenhang mit Ereignissen oder Willenshandlungen. In Wundts Gefühlstheorie galten sie neben den Dichotomien Lust-Unlust und Erregung-Hemmung als eine Dimension der Gefühle, die deren Zeitcharakter erfassen sollte. ${ }^{24}$

\section{Trieb und Affekt: Gerichtete Spannung}

Das Spannungskonzept der physiologischen Psychologie, das Gefühle auf körperliche Prozesse zurückführte, fand auch in die Nachbardisziplinen Eingang. In

21 Patrick Kury: Der überforderte Mensch. Eine Wissensgeschichte vom Stress zum Burnout. Frankfurt a. M./New York 2012, S. 37-54; vgl. auch Hans-Georg Hofer: Nervenschwäche und Krieg. Modernitätskritik und Krisenbewältigung in der österreichischen Psychiatrie 1880-1920. Wien 2004; Roelcke: Krankheit (wie Anm.12), S. 101-111; Joachim Radkau: Die wilhelminische Ära als nervöses Zeitalter, oder: Die Nerven als Netzwerk zwischen Tempo- und Körpergeschichte. In: GG 20 (1994), S. 211-241, v. a. S. 212-216; Joachim Radkau: Das Zeitalter der Nervosität. Deutschland zwischen Bismarck und Hitler. München 2000.

${ }^{22}$ Mai Wegener: „Das Gefühl Liebe entspräche einer rechtsdrehenden Spiralbewegung der Hirnmoleküle“. Zur Figur des Psychophysischen Parallelismus im ausgehenden 19. Jahrhundert. In: Caroline Welsh/Stefan Willer (Hg.): „Interesse für bedingtes Wissen“. Wechselbeziehungen zwischen den Wissenskulturen. München 2008, S. 131-152.

23 Wilhelm Wundt: Grundzüge der physiologischen Psychologie, Bd. 2,4. Leipzig 1893, S. 275.

${ }^{24}$ Ders.: Bemerkungen zur Theorie der Gefühle. In: Philosophische Studien 15 (1900), S. 149-182. 
der Psychiatrie reicherte es sich den bestehenden Bedeutungsschichten von Spannung an, wobei weitgehend unklar blieb, ob beziehungsweise wie die verschiedenen Ebenen miteinander zusammenhingen. Auf diese Weise erweiterte sich das Bedeutungsspektrum des Begriffs Spannung um die Jahrhundertwende erneut.

Der österreichische Psychiater Richard von Krafft-Ebing beispielsweise verwendete den Begriff Spannung 1888 in drei Bedeutungszusammenhängen. Zum einen benutzte er den Ausdruck, um einen objektiv erhebbaren somatischen $\mathrm{Zu}$ stand oder ein subjektives Gefühl körperlicher Spannung zu beschreiben, etwa wenn eine Patientin über „Spannung im Leibe“ klagte. ${ }^{25}$ Zum anderen sprach Krafft-Ebing in seinem Lehrbuch von "psychischer Spannung“. Da er dabei auf physikalische Begriffe zurückgriff, wird implizit ein Zusammenhang zwischen elektrischer, körperlicher und psychischer Spannung hergestellt. Um zu zeigen, dass bei gewissen Melancholikern die „Lösung der psychischen Spannung“ „gehemmt" sein könne, beschrieb Krafft-Ebing einen Patienten, dem es nicht gelinge, eine "gewünschte Bewegung" auszuführen, obwohl er alle seine Kräfte anspanne. ${ }^{26}$ Schließlich brachte er Spannung mit Affekt in Verbindung. Ein Affekt werde, so der Psychiater, dann „besonders heftig“, wenn er sich zu einem „Streben“ entwickle und dieser „Spannungszustand keine sofortige Lösung“ finde. Eine "plötzliche Lösung der Spannung“, eine „Realisierung des Strebens“ rufe hingegen „einen Lustaffekt" hervor. ${ }^{27}$

Spannung wird hier als Kraft zwischen zwei Polen verstanden - ähnlich wie bei einer Redoxreaktion in einem galvanischen Element, wo die Spannung, die Differenz der Elektrodenpotentiale, die Triebkraft der Reaktion darstellt. Solange sich eine Spannung messen lässt, befindet sich die Reaktion nicht im Gleichgewicht. Krafft-Ebing übertrug also das Konzept der elektrischen Spannung auf die emotionale Ebene und ging davon aus, dass Spannungen stets nach einer Lösung strebten. Auf diese Weise postulierte der Psychiater einen Zusammenhang von Spannung und Triebkraft.

Auch Sigmund Freud kombinierte physikalische Begriffe wie Hemmung mit psychologischen Begriffen wie Lust oder Wunsch, verband nun aber Spannung mit Erregung. Nach Freud führten Triebe und Sinneseindrücke zu übermäßiger Erregung und damit zu einem Gefühl von Spannung. Da eine Erhöhung von Spannung mit Lust und eine Verminderung von Spannung mit Unlust einhergehe, zielten alle psychischen Vorgänge darauf ab, Spannung abzuführen. ${ }^{28}$ In den 1920er Jahren kam Freud zwar zu dem Schluss, „dass es auch lustvolle Spannungen und unlustige Entspannungen" gebe ${ }^{29}$ - ein Aspekt, der in der Kunst, in der Ästhetik und in der Alltagssprache schon lange eine wichtige Rolle spielte, finden

${ }^{25}$ Richard von Krafft-Ebing: Lehrbuch der Psychiatrie auf klinischer Grundlage für praktische Ärzte und Studirende. Stuttgart ${ }^{3} 1888$, S. 339, S. 452, S. 644.

${ }^{26}$ Ebd., S. 100.

27 Ebd., S. 17.

28 Sigmund Freud: Drei Abhandlungen zur Sexualtheorie. Leipzig/Wien 1905.

29 Ders.: Das ökonomische Problem des Masochismus. In: Internationale Zeitschrift für Psychoanalyse 10 (1924), S. 121-133, hier: S. 122. 
sich doch etwa in der Literatur neben „ängstlich“ durchaus auch „freudig gespannte" Helden und Heldinnen. ${ }^{30}$ Spannung besitze, wie Karl Büchler 1908 im Aufsatz „Die ästhetische Bedeutung der Spannung“ schrieb, einen ambivalenten „Gefühlston“, weshalb sie als „Mittelding von Erwartung und Zweifel, Wunsch und Furcht, Ungeduld und Neugier" beschrieben werde. ${ }^{31}$ In der Medizin blieb jedoch die Gleichsetzung von Spannung und Unlust wegweisend.

Was nämlich, wenn Spannung nicht gelöst werden konnte? Nach Freuds Neurosenlehre führten unbefriedigte sexuelle Bedürfnisse zu einer „Steigerung der psychischen Spannung“ und wirkten deshalb „pathogen“. „Es gibt nur zwei Möglichkeiten“, so Freud, „sich bei anhaltender realer Versagung der Befriedigung gesund zu erhalten, erstens, indem man die psychische Spannung in tatkräftige Energie umsetzt, welche der Außenwelt zugewendet bleibt und endlich eine reale Befriedigung der Libido von ihr erzwingt, und zweitens, indem man auf die libidinöse Befriedigung verzichtet, die aufgestaute Libido sublimiert und zur Erreichung von Zielen verwendet, die nicht mehr erotische sind und der Versagung entgehen." 32

Um Trieb mit Unlust zu verknüpfen, musste man allerdings Freuds Theorien nicht kennen. Bereits die „Allgemeine Realencyclopädie“ von 1849 definierte Trieb als „die allgemeine innere Bedingung des Strebens, vermöge welcher das Gemüth durch Lust und Unlust zu gewissen Arten des Handels angereizt" werde. ${ }^{33} \mathrm{Ab}$ Ende des 19. Jahrhunderts brachten die deutschsprachigen Lexika Trieb nicht mehr mit dem Gemüt, sondern mit Emotionen in Verbindung. Im „psychologischen Sinne“ bezeichne Trieb, so heißt es in „Meyers Konversations-Lexikon“ von 1908, die „Tendenz gewisser Gefühlszustände, sich unmittelbar in zweckmäBige, d. h. solche Bewegungen umzusetzen, welche geeignet sind, ein vorhandenes Unlustgefühl zu beseitigen, bez. ein Lustgefühl zu erzeugen “. ${ }^{34}$ In Kombination mit den Begriffen Trieb, Affekt oder Gefühl erhielt Spannung also eine stark dy-

30 Vgl. z. B. Theodor Mommsen: Römische Geschichte. Bd. 2: Von der Schlacht bei Pydna bis auf Sullas Tod. Leipzig 1855, S. 402: „Die entsetzlichen Krisen einer funfzigjährigen Revolution, das instinctmässige Gefühl, dass der Bürgerkrieg noch keineswegs am Ende sei, steigerten die angstvolle Spannung, die trübe Beklommenheit der Menge."; Lou Andreas-Salome: Fenitschka. Eine Ausschweifung. Stuttgart 1898, S. 176: „Meiner Mutter Stimme klang ängstlich gespannt.“; Johanna Spyri: Heidi's Lehr- und Wanderjahre. Gotha 1880, S.118: „,Willst du eins haben?‘ fragte der Thürmer, der Heidi’s Freudensprüngen vergnügt zuschaute. ,Selbst für mich? für immer?‘ fragte Heidi gespannt und konnte das große Glück fast nicht glauben."; Friedrich Theodor von Vischer: Auch Einer. Eine Reisebekanntschaft, Bd. 2. Stuttgart u. a. 1879, S.79: „Hilario tritt auf, heiter gespannt."

31 Karl Büchler: Die ästhetische Bedeutung der Spannung. In: Zeitschrift für Ästhetik und allgemeine Kunstwissenschaft 3 (1908), S. 207-254, hier: S. 210.

32 Sigmund Freud: Über neurotische Erkrankungstypen (1912). In: ders.: Gesammelte Werke, Bd. 8. Frankfurt a. M. 2001, S. 322-330, hier: S. 323.

33 Trieb. In: Allgemeine Realencyclopädie oder Conversationslexicon für das katholische Deutschland, Bd. 10. Regensburg 1849, S. 236.

34 Trieb. In: Meyers Großes Konversations-Lexikon. Ein Nachschlagewerk des allgemeinen Wissens, Bd. 19. Leipzig/Wien ${ }^{6} 1908$, S. 707; Ute Frevert u. a.: Gefühlswissen. Eine lexikalische Spurensuche in der Moderne. Frankfurt a. M. 2011, S. 107-113. 
namische Komponente. Triebe wurden mit Unlust verbunden und mussten deshalb befriedigt oder in die richtige Bahn gelenkt werden.

Starke Resonanz fand der Konnex von Trieb und Spannung an der Wende vom 19. zum 20. Jahrhundert im Sexualitätsdiskurs. Die österreichische Schriftstellerin Grete Meisel-Hess etwa, die für Sexual- und Sozialreformen und die Rechte der Frau eintrat, schrieb 1909, „unausgelöste[...] Spannungen“ - also unerfülltes geschlechtliches Begehren - würden die „Schaffenskraft“ des Individuums „erdrücken“, wobei sich eine mangelnde „Auslösung geschlechtlicher Spannung“ bei Frauen besonders negativ auswirke: „Gerade für die Frau ist ein befriedigendes Liebesleben vielleicht noch notwendiger als für den Mann, denn sie ist der aufnehmende Teil; aus dem, was ihr von ihm kommt, baut sie ihre Energien. Der Mann, als der Ausgebende, kann sich durch ein letztes Rettungsventil, das ihm die Natur gelassen hat und das ihm zur Entladung seiner Spannungen verhilft, befreien. “35

Dieser „natürliche“ Geschlechtstrieb, der sich durch heterosexuelle Handlungen befriedigen ließ, wurde klar von einem sexualpathologischen Geschlechtstrieb unterschieden, den die Psychiatrie seit den späten 1860er Jahren zu erfassen suchte. Schon Wilhelm Griesinger hatte sich bemüht, den „Drang“ zu gleichgeschlechtlichen Handlungen und dessen Verhältnis zum Geschlechtstrieb zu klären. In Krafft-Ebings Fallgeschichten von 1882 verschmolzen der Geschlechtstrieb und der pathologische Trieb schließlich zum homosexuellen Trieb. „Mit der so entdeckten Homosexualität entstand ein neuartiger Geschlechtstrieb, der Sexualtrieb, der nun entweder homosexuell und damit pathologisch oder physiologisch und damit heterosexuell war.“36

Der pathologische Sexualtrieb galt als eine autonome Kraft, die starken Druck und damit ein hohes Maß an Spannung hervorrief. Magnus Hirschfeld erwähnt in seinem Lehrbuch von 1921 beispielsweise einen Pädophilen, der berichtete, er versuche seine „Erregtheit und Spannung“, die zu einer schweren „Unruhe“ führten, durch „gute Musik“ und „schnelle[s] Fahren“ im D-Zug „wenigstens einigermaßen [zu] betäuben“. Und eine Frau klagte, sie habe wegen ihrer „fortwährenden Spannung“ unter „Weinkrämpfe[n]“ und „Perioden exaltierter Lustigkeit oder Wochen stumpfer Melancholie“ gelitten. ${ }^{37}$

Die Denkfigur, dass Spannung immer nach einer Lösung dränge und auf Dauer der psychischen Gesundheit schade, ließ sich gut mit der Vorstellung vereinbaren, dass psychische Störungen häufig mit einem hohen Maß an Spannung verbunden seien. Weit öfter als von einem Mangel an Spannung war deshalb in der Psychiatrie ab der Jahrhundertwende von einer „gehemmten“ oder „hohen“ Spannung die

35 Grete Meisel-Hess: Die sexuelle Krise, Jena 1909. In: Mark Lehmstedt (Hg.): Deutsche Literatur von Frauen. Berlin 2001, S. 48966-49535, hier: S. 49110.

36 Philippe Weber: Der Trieb zum Erzählen. Sexualpathologie und Homosexualität, 1852-1914. Bielefeld 2008, S. 37, S. 43.

37 Magnus Hirschfeld: Sexualpathologie. Ein Lehrbuch für Ärzte und Studierende. 1. Teil: Geschlechtliche Entwicklungsstörungen mit besonderer Berücksichtigung der Onanie. Bonn ${ }^{2} 1921$, S. 54, S. 274. 
Rede, die früher oder später gewaltsam durchbreche. In Bleulers „Lehrbuch der Psychiatrie“ von 1916 heißt es beispielsweise, in Affekten liege eine „Tendenz zum Handeln“, die „oft von innen oder von außen [...] unterdrückt" werde. In der dritten Auflage des Lehrbuchs von 1920 ist dann von einer „Triebkraft“ der Affekte die Rede, die sich mit einer „physikalischen Energiespannung“ vergleichen lasse. Könne diese Triebkraft nicht in Handlung umgesetzt werden, bleibe sie mit ihrer „Spannung“ bestehen. Erfahre man „neue Antriebe zu dem gleichen oder nur ähnlichen Handeln“, verstärke sich die Spannung, „so daß sie unter Umständen schließlich explosionsartig alle Hindernisse überwindet und eventuell sogar wie ein gehemmter Reflex intensiv und extensiv über das Ziel hinausschießt“. Besonders bedrohlich schien, dass pathologische Spannungszustände „allerlei Symptome hervorbringen“ konnten, „ohne an Energie einzubüßen“, mithin immer neue „Explosionen“ befürchtet werden mussten. ${ }^{38}$

\section{Die soziale Dimension affektiver Spannung}

Ab dem Ende des 19. Jahrhunderts litten beispielsweise nicht nur Neurotiker und Epileptikerinnen unter Spannungszuständen, sondern auch Homosexuelle, Psychopathinnen und Schwachsinnige - Personen mit Diagnosen also, die zeitgenössische Psychiater einem Grenzbereich zwischen Normalität und Krankheit zuordneten. Gerade bei diesen Diagnosen zeigt sich deutlich, dass das Spannungskonzept der Psychiatrie einen eminent bürgerlichen Charakter hatte. Dem Ideal eines emotionalen Gleichgewichts entsprechend, verstand die Psychiatrie das Affektleben als ständigen Kampf zwischen Trieben, Emotionen und einer inneren Kontrollinstanz. Soziale Normverstöße erfolgten dann, so die Vorstellung, wenn es dieser Widerstandskraft nicht gelang, Triebe und Emotionen zu regulieren. ${ }^{39}$ Eine fehlende Trieb- und Affektkontrolle führte also nicht mehr einfach zu einer Störung eines idealen Gleichgewichts und zur Krankheit wie in früheren Konzepten. In einem hydraulischen Emotionskonzept, wie es schon Krafft-Ebing vertrat, reguliert sich der „psychische Apparat“ selbst: Wird an der einen Stelle etwas unterdrückt, kommt es früher oder später an anderer Stelle wieder hoch und entlädt sich.

In der forensisch-psychiatrischen Praxis kam der Konnex von Spannung, Trieb und Affekt besonders bei den Diagnosen Schwachsinn und Psychopathie zum Tragen. Emil Kraepelin etwa schrieb in seinem Lehrbuch von 1915, bei „erregbaren Psychopathen“ entlade sich die „innere Spannung vor allem in Willkürhandlungen“. 40 Die mangelnde Trieb- und Affektkontrolle, die sich in „Reizbar-

38 Eugen Bleuler: Lehrbuch der Psychiatrie. Berlin 1916, S. 27; ders.: Lehrbuch der Psychiatrie. Berlin/Göttingen/Heidelberg ${ }^{3} 1920$, S. $27-29$.

39 In der Psychiatrie wurden die Begriffe Affekt und Gefühl in der Regel nicht definiert und meist synonym verwendet.

${ }^{40}$ Emil Kraepelin: Psychiatrie. Ein Lehrbuch für Studierende und Ärzte. Leipzig 81915, S. 1992 f. In einem anderen Kontext konnte Kraepelin Spannungszustände allerdings durchaus positiv beurteilen: „Die durch Spannung der Erwartung erreichte Verschärfung des successiven Contrastes 
keit“, „Impulsivität“ oder „Haltlosigkeit“ manifestierte, wurde bei Psychopathen und Schwachsinnigen nicht auf eine eigentliche Geisteskrankheit, sondern auf eine pathologische Unfähigkeit zurückgeführt, die primär als angeboren galt. ${ }^{41}$

Obwohl Geisteskrankheiten grundsätzlich auch mit einem Mangel an Spannkraft verbunden sein konnten, ${ }^{42}$ meinte Spannung in der Psychiatrie um 1900 also meist starke oder zu starke Spannung. Geisteskranke und „Anormale“, denen eine hohe Triebhaftigkeit zugeschrieben wurde, konnten - so die Vorstellung - ihre Affekte nicht oder zu wenig regulieren und litten deshalb unter einer außergewöhnlich starken Spannung, die sich früher oder später explosionsartig entlud. Diese Denkfigur verdichtete sich schließlich im Fachbegriff der affektiven Spannung, der in den 1940er Jahren entstand. Affektive oder emotionale Spannung, wie man später auch sagte, galt als Symptom und hing immer mit einer psychischen Störung zusammen. ${ }^{43}$

So heißt es beispielsweise in einem psychiatrischen Gutachten aus dem Jahr 1940, der Patient leide unter einer „krankhaft[en]“ „Veränderung der Affektivität“, seine „Reizschwelle“ sei tiefer „als bei Normalen“. Er verberge „unter seiner zur Schau getragenen Gemütsruhe und Liebenswürdigkeit im Umgang eine Spannung“, „die oft während längerer Zeit völlig latent“ sei, „periodisch“ aber so stark werde, dass sie „nach aussen“ durchbreche und sich in „planloser Zerstörungswut und Gewalttätigkeit“ manifestiere. Der begutachtende Arzt stellte also ein pathologisches Missverhältnis zwischen äußeren Reizen und Gefühlsreaktion fest, eine quantitative Abweichung, die an der Norm eines inneren Gleichgewichts gemessen wurde. Er stellte die Diagnose Psychopathie und empfahl, den Mann zu bevormunden und vorerst ,in einer geschlossenen Anstalt“ zu belassen. ${ }^{44}$

Der Hinweis auf die versteckte Spannung des Patienten macht deutlich, dass der Gutachter von der Existenz latenter Spannungen ausging, die sich nicht ohne Weiteres erkennen ließen. Unterschwellige Spannung spielte in der Psychiatrie eine wichtige Rolle. Aus Publikationen und Krankenakten geht hervor, dass Ärzte meist aufgrund von Indizien wie Mimik oder Gestik auf "Spannungszustände“ schlossen. In der psychiatrischen Anstalt war Spannung ein Schlüsselbegriff. Be-

bezeichnet man als ,Pointe'. Sie bedeutet das unvermittelte Aufeinanderplatzen unvereinbarer Vorstellungen, die plötzliche paradoxe Lösung eines psychischen Spannungszustandes.“; Emil Kraepelin: Zur Psychologie des Komischen. In: Wilhelm Wundt (Hg.): Philosophische Studien, Bd. 2. Leipzig 1885, S. 128-193, S. 327-369, hier: S. 360.

${ }^{41}$ Urs Germann: Psychiatrie und Strafjustiz. Entstehung, Praxis und Ausdifferenzierung der forensischen Psychiatrie in der deutschsprachigen Schweiz 1850-1950. Zürich 2004, v. a. S. 259-276. ${ }^{42}$ Bei Pierre Janet ermöglichte psychische Spannung die psychische Synthese. Störungen traten auf, wenn die Spannung vermindert war. Eugen Bleuler nahm Janets Konzept auf und postulierte, dass Schizophrenie zu einer Lockerung der Assoziationsspannung führe. Heinz Schott/Rainer Tölle: Geschichte der Psychiatrie. Krankheitslehren, Irrwege, Behandlungsformen. München 2006, S. $98 \mathrm{f}$.

${ }^{43}$ Vgl. dazu Marietta Meier: Spannungsherde. Psychochirurgie nach dem Zweiten Weltkrieg. Göttingen 2015.

${ }^{44}$ Psychiatrische Klinik Rheinau, KA-Nr. 9702, S. 10f., Abschrift des Gutachtens der Psychiatrischen Anstalt Realta vom Oktober 1940. 
merkungen wie „ruhig[,] aber gespannt“, „macht unvertrauten, gespannten Eindruck“ oder „zeitweise unheimliche Gespanntheit“45 zeigen, dass das Klinikpersonal „gespannte“ Patienten für belastend und gefährlich hielt, weil man fürchtete, dass sich Spannungen jederzeit „entladen“ könnten. Um solche „Ausbrüche“ zu verhindern oder möglichst schnell einzudämmen, wurden die Patientinnen deshalb aufmerksam beobachtet.

Worin „affektive Spannung“ aus medizinischer Sicht auch immer gründete - ab dem ausgehenden 19. Jahrhundert erhielt Spannung in der Psychiatrie eine soziale Bedeutungsebene. Das hydraulische Emotionskonzept nährte die Furcht des Bildungsbürgertums vor einem individuellen und kollektiven Kontrollverlust. ${ }^{46} \mathrm{Ob}$ es um eine „gespannte“ Patientin ging, um die Begutachtung eines Straftäters oder um gesellschaftspolitische und weltanschauliche Fragen - Spannungszustände konnten jederzeit kippen und eine unheilvolle Dynamik annehmen.

Bei affektiver Spannung drohte daher stets Gefahr; eine Gefahr, deren Ursachen, Anzeichen und Ausmaß, so der Anspruch, nur die Psychiatrie beurteilen konnte. Dass der instabile Zustand in einen destruktiven Prozess übergehen würde, verstand sich offenbar von selbst oder musste zumindest in Erwägung gezogen werden, konnten doch Spannungsentladungen für Individuen, soziale Gruppen und ganze Gesellschaften schwerwiegende Folgen nach sich ziehen. In der Psychiatrie hatte Spannung deshalb viel gemein mit einem Zustand gestörten Einvernehmens - eine Begriffsbedeutung, die beispielsweise in der Soziologie oder der Geschichtswissenschaft üblich war. ${ }^{47}$ Ging es aber jenen Disziplinen in erster Linie darum, Spannungen zu beschreiben und zu erklären, sollte die Psychiatrie auch verhindern, dass Spannungen eskalierten. Dieser Anspruch blieb zunächst auf die individuelle Ebene beschränkt, betraf nach 1900 jedoch auch gesellschaftspolitische Fragen. In „The Neuroses of the Nations“ zum Beispiel, einer sozialpsychologischen Studie aus dem Jahr 1925, wurde der Erste Weltkrieg auf „eine psychische und moralische Störung“ zurückgeführt, die die Krieg führenden Länder erfasst und in ganz Europa „Spannung und Angst“ ausgelöst habe. Das Buch wollte deshalb einen Beitrag zu einer „präventiven Psychiatrie“ leisten, die in Zukunft helfen könne, ähnliche Katastrophen zu verhindern. ${ }^{48}$

\footnotetext{
45 Staatsarchiv des Kantons Zürich, Z100, Krankenakten Psychiatrische Universitätsklinik Zürich, KA-Nr.27603, S.14, Eintrag vom 15.8.1940; Psychiatrische Klinik Waldhaus, KANr. 17536, S. 8, Eintrag vom 3. 9. 1947; Psychiatrische Klinik Rheinau, KA-Nr. 9556, Schreiben an den Amtsvormund des Patienten, 18.2.1950.

46 Vgl. Germann: Psychiatrie und Strafjustiz (wie Anm. 41), S. 276.

47 Vgl. bspw. Georg Simmel: Philosophie des Geldes. Leipzig 1900, S. 542: „Durch die moderne Zeit, insbesondere, wie es scheint, durch die neueste, geht ein Gefühl von Spannung, Erwartung, ungelöstem Drängen - als sollte die Hauptsache erst kommen, das Definitive, der eigentliche Sinn und Zentralpunkt des Lebens und der Dinge.“

${ }^{48}$ Carolyn E. Playne: The Neuroses of the Nations. London 1925; E. E. Sperry: The Neuroses of the Nations. Review. In: AHR 31 (1925), S. 137f.; vgl. auch den Beitrag von Thomas Beddies in diesem Band.
} 


\section{Fazit: Spannung und Macht}

Spannung konnte an der Wende vom 19. zum 20. Jahrhundert vieles meinen: mechanische, elektrische oder körperliche Spannung, soziale, politische, innere oder psychische Spannungen. Der Begriff wurde in zahlreichen Bereichen und Zusammenhängen verwendet, wobei sich die verschiedenen Bedeutungen oft nicht klar voneinander trennen ließen, sondern ineinander überflossen. Als boundary concept ermöglichte der Ausdruck Spannung deshalb der Psychiatrie, an alltagssprachliche Vorstellungen von Spannung anzuknüpfen und diese mit Begriffen und Wissensbeständen verschiedener Fachdisziplinen, vor allem der Physiologie, der Neurologie und der Psychologie, zu verbinden.

War in der Psychiatrie von Spannung die Rede, konnten dem Begriff also ganz unterschiedliche Spannungskonzepte zugrunde liegen. Dass sich diese Vorstellungen kaum auf einen gemeinsamen Nenner bringen ließen, spielte in der Regel keine Rolle. Jeder wusste: Spannungen konnten nicht nur krankhaft sein, sondern auch krank machen. Spannung war deshalb in der Psychiatrie kein Begriff, der definiert und diskutiert wurde. Die Wirksamkeit, die der Ausdruck entfaltete, gründete vielmehr in seiner Langlebigkeit, seiner Unscheinbarkeit und seinem breiten semantischen Feld. Da mit Spannung Körperfunktionen, Nerven, Triebe, Affekte, Emotionen und deren soziale Folgen assoziiert werden konnten, ermöglichte der Begriff, Körper und Geist, Individuum und Gesellschaft miteinander in Verbindung zu bringen, ohne klären zu müssen, wie diese verschiedenen Ebenen zusammenhingen. ${ }^{49}$

Das Spannungskonzept, das die Psychiatrie in der langen Jahrhundertwende vertrat, zeichnet sich durch eine eigentümliche Hybridität aus: Es beruhte einerseits auf der Vorstellung, dass der Körper ein selbstregulierender Organismus sei, in dem dynamische Vorgänge wirkten, die ein innerorganisches Gleichgewicht anstrebten. Auf der anderen Seite ging man davon aus, dass Geisteskranke und "Abnormale“, unter Umständen sogar soziale Gruppen und ganze Gesellschaften Spannungen nicht regulieren könnten oder diese auf eine Art und Weise regulierten, die sie und andere in Gefahr bringe. Um Sicherheit zu gewährleisten und Konflikte zu verhindern, musste in solchen Fällen von außen eingegriffen werden, also eine andere Form von Dynamik zum Zug kommen. War in der Psychiatrie von Spannung die Rede, ging es daher immer auch um Macht.

Karl Jaspers weist in seinem Überblick zur Psychopathologie von 1946 darauf hin, dass Spannung in allen Bereichen des Lebens eine zentrale Rolle spiele: „Span-

${ }^{49}$ In dieser Hinsicht gleicht Spannung dem Begriff Stimmung, den Caroline Welsh untersucht hat. Die Figur der Stimmung findet sich zwischen 1750 und 1850 in der Anthropologie, Physiologie, Psychologie, Ästhetik, Literatur und Musik, wo sie je nach Kontext unterschiedliche Funktionen übernahm. Vgl. zum Beispiel Caroline Welsh: Stimmung. The Emergence of a Concept and its Modifications in Psychology and Physiology. In: Birgit Neumann/Ansgar Nünning (Hg.): Travelling Concepts for the Study of Culture. Berlin/New York 2012, S. 267-289. Ähnliche Studien gibt es auch zu den Begriffen Hemmung, Aktion und Reaktion: Roger Smith: Inhibition. History and Meaning in the Science of Mind and Brain. London 1992; Jean Starobinski: Aktion und Reaktion. Leben und Abenteuer eines Begriffspaars. Wien 2001. 
nung und Entspannung ist eine vom Biologischen bis zur Seele und zum Geist gehende Polarität. Sie geht vom Muskel über den Willen bis zur weltanschaulichen Grundhaltung. Was aber als physiologisches Geschehen natürlicherweise in rhythmischem Wechsel zum Gleichgewicht führt, das wird im Seelischen aus einem bloßen Geschehen zur Aufgabe. " 50 Was jedoch - so die Frage, die in der Psychiatrie stets im Raum stand -, wenn diese Aufgabe nicht bewältigt, die Spannung zu stark wurde, überhand nahm und ihr nichts mehr standhalten konnte?

\section{Abstract}

Tension (Spannung) is defined as a state of affairs hanging in the balance - a static, yet unstable state on the fringes of productive or destructive processes. The term's usage in the German language has been documented as early as the $17^{\text {th }}$ century. At that time, analogously to the transitive usage of the verb 'to tense', the term referred exclusively to the action of tightening something or to being tightened: horses were and are yoked, bows drawn, strings tautened. From the $18^{\text {th }}$ century onwards tension also signified a state of strained relations, and finally, from the $19^{\text {th }}$ century, the psychological condition of a person who was anticipating something, whose attention was stirred up. At the same time, a new term was coined with the invention of electric tension. With the establishment of physiology as a discipline since the mid-19th century, the nervous system itself was increasingly viewed as a system of electric impulses, nerve pathways, and energy flux. Simultaneously, every-day experiences were increasingly interpreted in such categories: thus the effect modern cities took on the individual was characterized as tension or overstimulation. In 1900 the term tension was thus employed in numerous contexts. The boundaries between the different definitions were expanded and blurred, translation and adoption processes can be discerned. It is the proposed article's goal to scrutinize the reciprocal effects as they took place between the different concepts of the term tension. In doing so, the psychiatric field plays a central role in the analysis.

In psychiatry, psychological tension has been linked with other, different forms of tension - in their texts, psychiatrists often hold the mechanistic view that, in order to restore an equilibrium, tension has to build and reach breaking-point. In contrast to other areas, the terms tension, tenseness, and state of stress were always defined in a distinctly negative way. Tension could not only make you sick, it could even become pathological, being closely related to madness or at least psychological alterity. Tension discharge could yield dangerous results. It is for this reason that, from the psychiatrists' point of view, a 'high affective tension' always poses a threat - a danger whose magnitude and causes can solely be assessed, so they claim, by themselves.

${ }^{50}$ Karl Jaspers: Allgemeine Psychopathologie. Berlin/Göttingen/Heidelberg ${ }^{4} 1946$, S. 286. 